\title{
Encouraging Equilibrium: Career-Life Balance Outreach and the Advancement of Women in Engineering
}

\author{
Autumn Reed, Ph.D. ${ }^{1}$, Renetta G, Tull, Ph.D. ${ }^{1}$ \\ ${ }^{1}$ University of Maryland Baltimore County (UMBC), USA, autumn2@umbc.edu, rtull@umbc.edu
}

\begin{abstract}
Issues related to career-life balance (CLB) disproportionally affect women in STEM. These issues disrupt women's career pathways, and in many cases, push them out of academia. In order to halt the exodus of women from academic careers in STEM, universities must develop interventions around CLB that recognize and address the everyday gendered CLB challenges that women graduate students, postdoctoral fellows, and faculty face. This paper showcases narratives from the University of Maryland, Baltimore County's (UMBC) international CLB initiative with underrepresented STEM faculty and graduate students that set the stage for the development of three additional CLB projects. The results of the former international project inform the partial implementation of the expansion projects at UMBC, which include: 1) Accelerating Post-Leave Associate Professor, Advancement through Intensive Support at Critical Junctions, 2) a campus-wide CLB awareness campaign and, 3) campus-wide CLB educational workshops. Through this outreachcentered paper, anchored in existing best practices and first person narratives of CLB struggles at UMBC, we aim to spur conversations and provide a model for other institutions to weave $C L B$ into the fabric of university culture as a normalized and cherished community value.
\end{abstract}

Keywords-Career-Life Balance, Women, Advancement

Digital Object Identifier (DOI): http://dx.doi.org/10.18687/LACCEI2015.1.1.085

ISBN: 13 978-0-9822896-8-6

ISSN: $2414-6668$

$1^{\text {th }}$ LACCEI Annual International Conference: “Engineering Education Facing the Grand Challenges, What Are We Doing?” July 29-31, 2015, Santo Domingo, Dominican Republic ISBN: 13 978-0-9822896-8-6

ISSN: 2414-6668

DOI: http://dx.doi.org/10.18687/LACCEI2015.1.1.085 


\title{
Encouraging Equilibrium: Career-Life Balance Outreach and the Advancement of Women in Engineering ${ }^{*}$
}

\author{
Autumn Reed, Ph.D. and Renetta G, Tull, Ph.D. \\ University of Maryland Baltimore County (UMBC), USA, autumn2@umbc.edu, rtull@umbc.edu
}

\begin{abstract}
Issues related to career-life balance (CLB) disproportionally affect women in STEM. These issues disrupt women's career pathways, and in many cases, push them out of academia. In order to halt the exodus of women from academic careers in STEM, universities must develop interventions around $C L B$ that recognize and address the everyday gendered $C L B$ challenges that women graduate students, postdoctoral fellows, and faculty face. This paper showcases narratives from the University of Maryland, Baltimore County's (UMBC) international CLB initiative with underrepresented STEM faculty and graduate students that set the stage for the development of three additional $C L B$ projects. The results of the former international project inform the partial implementation of the expansion projects at UMBC, which include: 1) Accelerating Post-Leave Associate Professor, Advancement through Intensive Support at Critical Junctions, 2) a campus-wide CLB awareness campaign and, 3) campus-wide CLB educational workshops. Through this outreach-centered paper, anchored in existing best practices and first person narratives of CLB struggles at $U M B C$, we aim to spur conversations and provide a model for other institutions to weave $C L B$ into the fabric of university culture as a normalized and cherished community value.
\end{abstract}

Keywords-Career-Life Balance, Women, Advancement

\section{INTRODUCTION}

Increasing the representation of women in engineering is a U.S. national priority, as evidenced by recent national conversations, including U.S. President Barack Obama's remark, "we need to have more girls interested in math, science, and engineering. We've got half the population that is way underrepresented in those fields and that means that we've got a whole bunch of talent...not being encouraged the way they need to" [1]. According to a 2013 American Society for Engineering Education report, women received $22.4 \%$ of engineering doctorates awarded in the United States and represent only $14.5 \%$ of tenure/tenure-track faculty positions [2]. The Institute of Electrical and Electronic Engineers explains how women "[face] gender bias, [have] few role models, and [lack] support from family and friends for their non-traditional career choice" [3]. These CLB challenges, coupled with issues of implicit bias and chilly departmental climate only intensify an already leaky pipeline for women in STEM.

Although we have made progress in partnership with such organizations as The Latin American and Caribbean Consortium of Engineering Institutions (LACCEI), The National Science Foundation ADVANCE Program (NSF), the Institute of Electrical and Electronic Engineers (IEEE), the American Society for Engineering Education (ASEE), and the Women in Engineering Proactive Network (WEPAN), additional efforts remain, in particular around the issue of Career-Life Balance (CLB). Literature suggests that women are forgoing faculty careers in engineering at an alarming rate due to the incompatibility of the rigorous demands of academia and other competing life interests and responsibilities, many of which are gendered and invisible [4]. Institutions must do more to vigorously foster climates that promote CLB in order to ensure women thrive at all stages in their engineering career. This paper showcases efforts at the University of Maryland, Baltimore County (UMBC), a midsize Carnegie rated doctoral granting, research-intensive institution, to promote CLB for women engineering graduate students, postdoctoral fellows, and faculty. We argue that it is imperative that universities embrace, model, and normalize CLB at all levels of the institution, transforming CLB into a cherished university value. This outreach-paper aims to encourage other institutions of higher education to engage in CLB conversations and enact meaningful CLB interventions.

\section{FIRST-PERSON NARRATIVES OF CLB STRUGGLES}

\section{A. Background}

In an effort to examine CLB issues that affect women faculty, we decided to develop an isolated context to observe phenomena. We chose entry and access to professional networks as our context. Filtering further, we focused on women's lack of international professional relationships, and ways that issues of career-life balance hinder development of

\footnotetext{
* This work is partially supported by The National Science Foundation (NSF), Grant \#1449322, Division of Engineering Education and Centers/ Broadening Participation in Engineering (BPE), and NSF Career-Life Balance Initiative - Grant \#1446406.
}

$1^{\text {th }}$ LACCEI Annual International Conference: “Engineering Education Facing the Grand Challenges, What Are We Doing?” 
global networks. Narrowing the scope once again, we further surmised that the international professional networks of women who are underrepresented in science, technology, engineering, and math (STEM) might be sparse. We hypothesized that women of color, or women from racial ethnic backgrounds that are underrepresented in the United States, African-Americans or Hispanic/Latinas in particular, are not participating in opportunities to build professional networks. The basis of this conjecture stems from anecdotal examples from conversations with underrepresented women faculty at such conferences and meetings as those featuring projects from NSF's ADVANCE: Increasing the Participation and Advancement of Women in Academic Science and Engineering Careers, and such meetings at annual conferences as the National Society of Black Engineers, the Society for Hispanic Professional Engineers, and with faculty Mentors-inResidence who participate in PROMISE: Maryland's Alliance for Graduate Education and the Professoriate (AGEP). The conversations reveal several reasons why women of color are not participating in opportunities to build international networks, including career-life balance. Therefore, we decided to develop a project that would specifically examine careerlife balance within the environment of an international experience.

In July 2014, we assembled a team of male and female graduate students, faculty, and staff from African-American and Hispanic/Latino backgrounds and immersed them in an international experience for one week. The group included participants drawn from three NSF programs associated with UMBC: The ADVANCE Hispanic Women in STEM project between UMBC and Puerto Rico, the PROMISE AGEP program with the University System of Maryland, and the UMBC Louis Stokes Alliance for Minority Participation Bridge to the Doctorate (LSAMP-BD) program. Through the ADVANCE program, we also connected with faculty from Jackson State University and North Carolina State University who were involved in programs that were specifically focused on faculty diversity, e.g., the ADVANCE program at Jackson State University in Jackson, Mississippi, USA, and the Office of Faculty Development and Special Initiatives Team in the College of Engineering at North Carolina State University (NC State), in Raleigh, North Carolina, USA. It was important to involve leaders from these initiatives because they were likewise bringing attention to the CLB struggles of minority women faculty in engineering. Our chief goal was to ascertain the thoughts of current U.S. women faculty in engineering and computer science, and future faculty- male and female graduate students on CLB. We included male students on the team based on experiences and lessons learned from the "Women in STEM" session at the World Engineering Education Forum (WEEF) in Cartagena, Colombia. At this event male undergraduate engineering students discussed their respect for their fellow women engineering peers because of exposure to issues about women in STEM, work experiences that involved teams with both genders and/or included women as team leaders. Furthermore, the "Women in STEM" sessions at the LACCEI conference 2013 (Mexico) and 2014 (Ecuador) included discussions about the need for "male champions" who, from their positions of power, would advocate for issues that involved women's access to professional opportunities and advancement. The team also included two social scientists to add cross-disciplinary thought to the conversations.

\section{B. Methods}

Fifteen participants were chosen from ADVANCE, LSAMP$\mathrm{BD}$, and the PROMISE AGEP-T program. Participants were invited to have an immersive experience in Guayaquil, Ecuador that would include an opportunity to mentor undergraduate engineering students at Escuela Superior Politécnica del Litoral (ESPOL) in Guayaquil, participate in the 2014 LACCEI conference with particular attention paid the "Mujeres en STEM y Diversidad" forum and the conference sessions that discussed women and diversity in engineering. Additional activities included informal cultural and scientific excursions in the region to assist with crosscultural communication and understanding. Twelve participants were able to attend the conference in person, and the additional participants joined in conversations online. Participants were given the following directions:

The study is designed to observe responses of participants from the University System of Maryland, NSF Louis Stokes Alliance for Minority Participation (LSAMP) Bridge to the Doctorate Fellows and Alumni, members of the NSF LSAMP community, graduate student members and faculty mentors from the NSF PROMISE AGEP community, and partners of the NSF ADVANCE Hispanic Women in STEM project who attend the Latin and Caribbean Consortium of Engineering Institutions annual conference in Guayaquil, Ecuador, and their reflections on the conference workshops. We are interested in their thoughts about career-life-balance. We will be asking them to record their thoughts using a blog. Upon return to the states, we will have follow-up discussions and webinars regarding the international experience and career-life-balance, and the general public will be invited to participate in the online discussions. This is a social media, interactive project. Men and women are invited to participate.

Questions were developed organically, based on the experiences that the faculty and graduate students were having in real-time during the meetings at ESPOL, on-site at the conference, and during the cultural experiences. Content for questions was drawn from conversations and lessons learned through the ADVANCE program, the PROMISE AGEP, and through our participation in conferences such as the Gender Summit 3 North America, Washington, DC, 2013.

13 $^{\text {th }}$ LACCEI Annual International Conference: "Engineering Education Facing the Grand Challenges, What Are We Doing?" July 29-31, 2015, Santo Domingo, Dominican Republic 
Six questions were posed, including some with multiple parts. A subset of the questions include the following:

1. Share your general thoughts over the next few days regarding your experiences with international collaborations, preparing for an international conference, observations as you travel, challenges, and expectations.

2. Discuss:

a) What did you learn from the Wednesday plenary and the Women in STEM and Diversity panels?

b) Is there a research benefit to meeting someone in person versus using technology to connect?

3. Discuss:

a) Has this trip facilitated any collaborations or research ideas that move you closer to your academic goals?

b) How can an excursion like this one contribute to career-life balance?

4. How does this photo convey collaboration and international engagement? (The question refers to a photograph of faculty and students sharing a meal and engaging in informal conversations.)

5. Discuss:

a) How did this international engagement experience influence your career strategies?

b) How will you encourage and model career-life balance for your current and future female mentees? (These questions referred to a talk on career-building strategies by Dr. Jaime Bonilla, of the Global Engineering Deans Council)

6. List:

a) 5 things that impede underrepresented graduate students and faculty in STEM from taking advantage of international research or collaboration opportunities.

b) 5 suggestions for increasing the numbers of underrepresented graduate students and faculty who will develop international collaborations.

c) 5 ways that international research and collaboration travel threatens or challenges the concept of career-life balance.

d) 5 ways that international research and collaboration travel can facilitate career-life balance.

All participants were required to blog daily. Participants were informed that excerpts from their comments may be used for research, and they were assured that no text would be added to their comments. They were informed that their online names may be used in the narrative for the research discussion, however, each participant was invited to use an existing online persona, use a pseudonym, or post as "Anonymous." All responses were considered equally valid. The combination of instructions, posted questions, and online responses yielded 186-posted entries for this project.

\section{Narratives}

The participants' blog responses from this project clearly encapsulate the daily dilemma that women in STEM face with regards to CLB. One participant, a female computer science faculty member, recalls an incident as a graduate student when

Once, I was asked to provide a schedule for when I was on campus so that a group meeting could be scheduled. I provided a schedule that did not include the times when I was taking care of my children. I was then told by a very senior professor that my schedule didn't look like I as a real graduate student. At the time I didn't even know how to defend myself against such a statement. Was my work getting done? Yes. Was I meeting the requirements of my program? Yes. Did he think I was progressing like a real graduate student before then? Yes. I honestly thought that if I had told him I was feeding the homeless during those times instead of taking care of my children, he would have thought differently of me.

This interaction suggests that "real" graduate students, who are taken seriously by their peers, are those students who are available 24/7. Moreover, this faculty member's narrative reveals her perception that notwithstanding her demonstrated success as a graduate student, her senior male professor did not value her responsibilities outside of the academy and effectively rendered this labor invisible and inconsequential. Most importantly, however, her account captures her lack of mentoring and support on coping with CLB issues as she "didn't even know how to defend [herself] against such a statement." Likewise, another current engineering graduate student participant laments how,

Males are "allowed" to focus solely on being breadwinners, and if their careers cause their domestic life to suffer, then it can almost be seen as commendable or a heroic sacrifice. However, a career female may risk a certain stigma if she does not have a clean house, well-manicured kids, and dinner on the table every night.

Read in tandem with the computer science faculty member's recollection, this graduate student's remarks point to the gendered double-bind that many women in STEM experience. The "real" graduate student the computer science faculty member describes is in reality, a category that is built from the socially constructed masculine role of "breadwinner." In other words, this "real" graduate student is a man. This gendered encoding of "real" graduate student ignores the power of the socially constructed feminine caregiving roles that are disproportionately assigned to women. As a field, engineering is not set up for women's success and women are written out of the script. Together, both examples demonstrate how our

$1^{\text {th }}$ LACCEI Annual International Conference: "Engineering Education Facing the Grand Challenges, What Are We Doing?" July 29-31, 2015, Santo Domingo, Dominican Republic 
conversations on CLB are disempowering and exacerbate the pressures and daily stresses that women engineers face in navigating gendered structural barriers that make achieving CLB all the more elusive.

There is also anecdotal evidence that women engineers at UMBC continue to struggle with CLB even as faculty. Women faculty both tenured and untenured have shared stories of how conflicts between their work and home responsibilities have caused them to fall short of their personal career goals in the form of unfunded labs, stifled research networks and collaborations, and publications. The consequences of this career-life imbalance are detrimental as external funding, publications, and national and international reputation are all central criteria for career advancement. Indeed, one non-engineering social scientist participant in the blogging project speculates,

If professors are already overloaded with the responsibility of teaching, research, and service plus, especially for women having to meet the demand of their family, what suffers in the process as individuals try to do it all...I've thought about staying in the academy after I graduate and there are a few things that make me think that perhaps this occupation might be asking too much. When I see professors, especially women work the second and third shift, often with little sleep, I wonder is it worth it? Am I willing to sacrifice my health and well-being to get tenure?

As these first-person testimonials demonstrate career-life balance remains a primary concern among women in engineering. This CLB imbalance, as demonstrated by the literature, has real costs associated for the women affected and the overall engineering pipeline. The gendered norms that circumvent open discussions about, mentoring around, and institutional interventions that foster CLB are deeply embedded in us as individuals, our institutions, and our language and cultural practices. The system is clearly broken. Acknowledging this rupture, the remaining sections of this paper discuss UMBC's successes, challenges, and current interventions to shift the campus-climate on CLB and empower women engineers in their quest for career-life equilibrium. The results from the narratives shared by the URM participants in the international experience provided qualitative data that is being used to inform the implementation of specific CLB interventions at the university level. In this "leap-frog" approach, we looked at the narrow issue of career-life balance within a broad context of developing international networks, and we are re-focusing the outcomes to inform UMBC's practice for CLB within academic departments for the College of Engineering and IT, and the College of Natural and Mathematical Sciences, and for the university at large.
III. UMBC: A MODEL FOR INCLUSIVE EXCELLENCE IN STEM

UMBC is recognized for preparing underrepresented minority (URM) students for careers in science and engineering through its Meyerhoff Scholars program and also for increasing the number and diversity of Ph.D. graduate who go on to academic careers through its NSF-funded PROMISE, Maryland's AGEP program. In addition to contributing to the diversity and success of URM students in STEM, as a result of its now institutionalized 2003 ADVANCE Institutional Transformation Grant and its 2012 PAID grant, UMBC is now a U.S. national leader in developing best practices to recruit, retain, and advance women and URM faculty in STEM. Many of UMBC's efforts around increasing the representation of women and URMs in STEM, engineering in particular have centered on addressing issues of CLB. The authors participate in a variety of initiatives at UMBC that are specifically geared toward increasing diversity at the graduate student, postdoctoral, and faculty levels.

\section{PROMISE: MARYLAND'S ALLIANCE FOR GRADUATE EDUCATION AND THE PROFESSORIATE (AGEP)}

PROMISE: Maryland's AGEP, sponsored by the National Science Foundation, is designed to increase the numbers and diversity of graduate students and postdoctoral fellows in STEM who will pursue the professoriate. The PROMISE AGEP: Maryland Transformation (AGEP-T) is a collaborative between UMBC, the University of Maryland College Park (UMCP), and the University of Maryland Baltimore (UMB), and it includes participation from all 12 institutions within the University System of Maryland. The PROMISE AGEP has a number of programs that are designed to recruit, cultivate, retain, and train underrepresented graduate students in STEM fields. PROMISE programs such as The Dissertation House, and the Summer Success Institute (SSI) have been pivotal interventions that contribute to degree completion and a sense of community that even involves members of students' families as stakeholders [5, 6, 7, 8, 9].

PROMISE AGEP's culture of mentoring, and dedication to STEM education on the part of all universities involved, has facilitated the process of degree completion. Notwithstanding this success, the program's plans to develop more professors have been met with challenges that include issues of careerlife balances. Anecdotal conversations, as well as qualitative data such as those obtained through the international project as described in Section II.C of this paper, reveal that some of the graduate students who participate in the PROMISE AGEP are concerned about pursuing the professoriate because they want to have families, and they are concerned about pursuing a career-path that they perceive does not value personal time. Students have heard horror stories about academic life, and these perceptions are influencing the career decisions of both URM men and women who are struggling with ways to utilize

13 $^{\text {th }}$ LACCEI Annual International Conference: "Engineering Education Facing the Grand Challenges, What Are We Doing?" July 29-31, 2015, Santo Domingo, Dominican Republic 
their research training, interest in teaching, and desire to live a full and balanced life.

One of our conjectures is that universities such as UMBC have policies in place that are supportive of needs such as maternity leave, however, graduate students and faculty are not taking advantage of them. We believe that there is a lack of awareness of policies, and fear of retribution if the policies are used. Given the data that we were gathering from projects such as the international engagement initiative in Ecuador, and other activities, UMBC is now working to implement a series of projects that will address issues of career-life balance for both graduate students and faculty, with particular emphasis on women, and with attention to URM women.

\section{UMBC-ADVANCE AND FAMILY FRIENDLY POLICIES}

The first step in developing the projects involved reviewing policies that were in support of career-life balance. In 2003, UMBC received a 3.2 million dollar ADVANCE Institutional Transformation Grant from the National Science Foundation. UMBC's development and implementation of a comprehensive Family Support Plan is a hallmark of the grant. The Family Support plan enables faculty members to create individual plans, which allow them to reduce or otherwise modify their workload, especially teaching duties to maintain a work/life balance while tending to a variety of family needs, including childbirth, adoption, eldercare, and family illness. This plan provides a 1-year tenure clock extension for pretenure faculty who have used the plan. The involvement of stakeholders from all levels of the institution in shaping the policy, and its endorsement by the highest levels of UMBC's senior administration, ensured the plan's successful normalization across campus. Most important, however, the plan functions as successful retention tool because the women STEM Assistant Professors who have used the plan have a high rate of tenure. As a measure of the plan's success, in 2012 the University System of Maryland adopted and institutionalized the plan for all 12 universities in the system. Notwithstanding the success of the Family Support Plan, UMBC realizes that works remains to tackle the more "invisible" aspects of family responsibilities that derail women's post-tenure promotion to full. Recent data shows that $54 \%$ of UMBC's women STEM associate professors have used the plan, but that they are not progressing to promotion to full at the same rate as their male counterparts.

\section{ON-RAMPS - A CLB INITIATIVE}

In 2014, UMBC received a 2-year EAGER grant from the National Science Foundation to fund "On-Ramps" to Full Professor: Institutional Support for Post-Family Leave Faculty Research Reintegration." This new pilot-initiative complements our on-going efforts to create an institutional climate that embraces CLB in the PROMISE and UMBCADVANCE programs. The "On-Ramps" initiative consists of three pilot projects 1) Accelerating Post-Leave Associate Professor, Advancement through Intensive Support at Critical Junctures, 2) Establishing a robust institutional CLB culture for graduate student, postdoctoral scholar, and faculty success, and 3 ) Eight seminars and community building workshops that promote CLB and create communities of practice.

\section{A. Project 1}

The "Accelerating Post-Leave Associate Professor, Advancement through Intensive Support at Critical Junctions" project is designed to address the relationship between "invisible" family responsibilities and the "unforgiving" lockstep and cyclical structure of academia, which requires constant external funding and publications. Promotion to full professor in engineering is dependent on successfully fulfilling both of these time-intensive activities. UMBC hypothesizes that women Associate Professors are not progressing to Full Professor at the same rate as their male counterparts due to productivity gaps that are directly related to the gendered and "invisible" CLB barriers women face. For the pilot project, UMBC identified a cohort of 16 women Associate Professors in rank 7 years or less, who accessed the Family Support Plan at any point in their faculty career to apply for an infusion of support in the form of course releases, graduate research assistant support, domestic and international travel funds, and research writing coach support. The 16 women were invited to submit research acceleration plans in which request the types of support that they believe will aid in their advancement to full. UMBC has funded 7 of the proposals for the 2015-2016 academic year and will offer such other forms of CLB support as workshops and writing groups to all 16 of the eligible associate professor women.

\section{B. $\quad$ Project 2}

The second project aims to establish a robust institutional culture for graduate student, postdoctoral scholar, and faculty success that "celebrates" CLB. UMBC will launch a campaign that will bring into focus the CLB policies that are already in place on campus, have a repository of resources, and showcase profiles (physical posters, and online) of faculty who are successfully balancing career and life. Our premise is that things that are done outside of UMBC do not have to remain secret. We will showcase the fact that people take the leave that they have earned to raise families, that they participate in health and wellness activities, and that they take time for community engagement. We will design and launch campus posters, banners, and have a web presence that will showcase this balance, and that will both celebrate and publicize the activities that represent a full life. Too often, time outside of the academy is stigmatized such that academics think that they will lose respect if they discuss vacations, or express excitement for activities that will be conducted outside of the

13 $^{\text {th }}$ LACCEI Annual International Conference: "Engineering Education Facing the Grand Challenges, What Are We Doing?" July 29-31, 2015, Santo Domingo, Dominican Republic 
lab. If faculty, postdocs, and graduate students know that "Career-Life Integration" benefits are extended to all, and if all are encouraged to partake of these benefits, we can continue to shift the culture.

\section{C. $\quad$ Project 3}

Our third project implements a series of campus-wide educational awareness programs that will include sharing of various opportunities for STEM workforce to accommodate family and work responsibilities, implicit bias workshops, and how UMBC policies and practices are aligned with the Office of Management and Budget's family-friendly policy guidance. The workshops seek to inform the campus by including topics on dual career support, CLB guidance from various federal agencies, and other efforts related to Title IX. In an effort to encourage CLB and promote productivity within community, we will also have 1-2 day workshops that bring members of the campus community (within their respective academic group, e.g., faculty, postdoc) to work on publications and grants. These workshops will include group coaching for the development of career/life integration plans for graduate students, postdocs and faculty. The premise is that we will develop communities to encourage productivity, while promoting CLB.

\section{CONCLUSION AND FUTURE WORK}

The identification of career-life balance as an issue that influences career decisions of women and URMs in STEM is a key to this work. If URM women graduate students who are being trained for the professoriate are considering opting out of the academy as a career, then the pipeline leaks further. If CLB is one of the issues that is a "perceptual barrier to entry" to the professoriate, then developers of programs such as NSF's AGEP and ADVANCE programs, which are geared toward developing and advancing URM and women STEM faculty, should take heed to address and break down the barrier. Some of narratives described in Section II.C describe situations where the benefits of the professoriate are perceived as being apportioned to men. In cases where men are perceived to be the sole beneficiaries of an academic career, women who have not seen examples of benefits in their favor, may choose to opt-out of the academy. UMBC is looking closely at issues of recruitment and retention of women faculty in engineering, and the university wants to create an environment that includes a culture that honors the integration of career and life, and one that will be both inviting and supportive of women from all backgrounds. Recognizing the importance of all of the members of the campus community, UMBC's initiative for career-life balance is attempting to ensure that women are not shut out of academic opportunities, and that their opportunities for creativity, innovation, and progress are not stifled. The development and implementation of these three projects are steps in a positive direction that can bring more attention to career-life balance as an issue that can influence faculty diversity, and advancement for women in STEM academic careers.

\section{ACKNOWLEDGMENT}

The authors acknowledge supportive actions and connection to initiatives from the following programs of the National Science Foundation: The Broadening Participation in Engineering Program, the NSF Career-Life Balance Initiative, PROMISE AGEP: Maryland Transformation (PROMISE: Maryland's Alliance for Graduate Education and the Professoriate - AGEP-T), The Louis Stokes Alliance for Minority Participation Bridge to the Doctorate Program (LSAMP-BD), and ADVANCE: Increasing the Participation and Advancement of Women in Academic Science and Engineering Careers. The authors particularly thank the ADVANCE Hispanic Women in STEM project between UMBC and Universidad Metropolitana (UMET) in Puerto Rico, which built the basis for other collaborations between UMBC and women engineering and computer science faculty at other universities in Puerto Rico. In addition to the NSF projects that are mentioned in the footnote on page 1 , the authors acknowledge synergistic activities sponsored by NSF PROMISE AGEP-T Grant \# 1309290. The following units and departments at UMBC also receive acknowledgment: Dr. Janet Rutledge, Dean of the Graduate School and Vice Provost for Graduate Education; and Dr. Patrice McDermott, Vice Provost for Faculty Affairs, Office of the Provost, UMBC's Faculty Diversity Initiatives. The following departments are also acknowledged for providing input during early discussions for the international project: UMBC's International Education Services Office, and The Shriver Center.

\section{REFERENCES}

[1] Obama, B. "Women in STEM." Office of Science and Technology Policy. 2014. [Online]. Available: http://www.whitehouse.gov/administration/eop/ostp/women, 2014.

[2] Yoder, B.L., "Engineering by the numbers." American Society for Engineering Education, 2014. [Online]. Available:

http://www.asee.org/papers-and-publications/publications/14_11-47.pdf, 2014.

[3] Pretz, K. "IEEE Women in Engineering Committee Celebrates 20 Years." The Institute.2014 [Online]. Available:

http://theinstitute.ieee.org/benefits/ieee-groups/ieee-women-inengineering-committee-celebrates-20-years

[4] Goulden, M., Frasch, K., and M. Mason. "Staying competitive: Patching America's leaky pipeline in the sciences." The Center for American Progressive, November 2009.

[5] Aparakakankanange, E., and Tull, R. G. An AGEP Program Analysis: Minority Graduate Student Diversity in STEM Disciplines at Three Universities. Proceedings of the 17th International Conference on Interactive Collaborative Learning (ICL2014)/2014 World Engineering Education Forum, Dubai, United Arab Emirates, 2014

[6] "About the Dissertation House," 2011. [Online]. Available: http://dissertationhouse.wordpress.com/about/

[7] Alliances for Graduate Education and the Professoriate, "AGEP," 2010 [Online]. Available: www.nsfagep.org. 
[8] Tull, R.G., Ordóñez, P., Carter-Johnson, F., Zayas, B., Byars-Winston, A. M. Cortes-Rodriguez, "The Jessica Effect: Valuing Cultural and Familial Connections to Broaden Success in Academe," AAC\&U Peer Review, 2014

[9] Tull, R. G., Rutledge, J.C., Warnick, J. W., and Carter, F.

D. (2012). PROMISE: Maryland's Alliance for Graduate Education and the Professoriate Enhances Recruitment and Retention of

Underrepresented Minority Graduate Students. Academic Medicine, $87(11)$, p. $1562-1569$.

13 $^{\text {th }}$ LACCEI Annual International Conference: "Engineering Education Facing the Grand Challenges, What Are We Doing?" July 29-31, 2015, Santo Domingo, Dominican Republic 